\title{
TWO-GROUP REACTOR NOISE ANALYSIS BY LANGEVIN'S TECHNIQUE
}

\author{
J. M. NiETo and P. AkhtaR \\ University of Michigan, Ann Arbor, Michigan, 48104 \\ and \\ N. J. ACKermanN, JR. \\ University of Tennessee, Knoxville, Tenn. 37902
}

(Received 1 July 1968)

\begin{abstract}
Langevin's technique is applied to two energy group space independent reactor model. Detection statistics and fluctuations in production and decay rate of delayed neutron precursors are also taken into account. Expressions for the auto- and cross-correlation functions of the fluctuating sources associated with various random processes such as fission, capture, scattering, etc., are obtained and used in finding the auto- and cross-power spectral densities of the detection rates in fast and slow groups. Numerical estimates for various break frequencies for a representative set of parameters are found.
\end{abstract}

\section{INTRODUCTION}

REACTOR noise has been the subject of extensive theoretical (COURANT et al., 1947; De Hoffman, 1949; Raievski, 1958; Harris, 1958; Klahr, 1958; Moore, 1958; Pal, 1958; Velez, 1959; Bennett, 1960; Dalfes, 1962; Osborn et al., 1963; BeLl, 1965; OtsuKa et al., 1965; OsBorN, 1965) and experimental investigations (ORNDOFF, 1957; BADGley et al., 1964; Trinko et al., 1965; NomURA, 1966; DraGt, 1966; GoTOH, 1966) in recent years. The keen interest displayed in the subject has been chiefly motivated by the possibility of obtaining useful information about macroscopic properties of reactors by analysing fluctuations in neutron distributions. In these investigations, Langevin's Technique (LANGEviN, 1908; LAX, 1960; CoHN, 1964; CoHN, 1960; AKCASU et al., 1966; SHEFF et al., 1966a; SHEFF et al., 1966b; SAITo, 1967a; SAITo, 1967b) has been frequently employed and generally one speed point reactor model has been used. The two energy group point model for critical reactors has been previously considered by COHN (1964) who has given relations between Fourier transforms of neutron density fluctuations in the two groups and the corresponding fluctuating sources normally known as noise equivalent sources (N.E.S.). More recently, SAITo (1967a) has applied Langevin's method to obtain the spectral matrix of the neutron fluctuations in a sub-critical space- and energydependent reactor model described by a vector whose components represent concentrations of particles (neutrons and precursors) in phase space points. In another paper, SAITo (1967b) derived the spectral matrix in the special case of one space group, one energy group and six-delay group model.

The present analysis extends the results of Cohn and Saito by: (1) including the detection process statistics (Section 3); (2) Obtaining explicit expressions for various N.E.S. (Section 4); and (3) obtaining explicit expressions for the power spectral densities showing the break frequencies (Section 5). In addition, a numerical application is included in Section 6. The general interest in investigating a two-energy group comes from the desirability of checking the adequacy of one group model, and to explore the possibility of obtaining any additional information from the autocorrelation functions of the fast group, as well as from its cross-correlation with the slow group.

In this work, it is assumed that the external current of the detectors is proportional 
to the instantaneous detection rate (AKCASU et al., 1966). The filtering effect of the detectors, arising mainly from the finite ion collection time, on the power spectral densities of the neutron density fluctuations, is thereby eliminated. Since this effect can be investigated separately (OsBorN et al., 1966), it is not included in the present theory.

Finally, in Appendix I, a general expression is derived for the spectral matrix of the external currents (AKCASU et al., 1966) of the detectors in a "multipoint reactor" model, with $n$ energy groups. For a definition of "multipoint reactor", the reader is referred to a paper by Saito (1967a).

\section{EQUATIONS DESCRIBING THE SYSTEM}

We consider a subcritical reactor operating at steady state. The reactor is described by a multipoint model consisting of two energy groups and $m$ types of delayed neutron precursors. For a generalization to $n$ energy groups and $m$ types of delayed neutron precursors, see Appendix I. Let $\left\langle N_{i}\right\rangle, i=1,2, \ldots, m+2=M$ denote the components of the macroscopic steady state vector $\langle\mathbf{N}\rangle$ describing the system. This vector satisfies the equation

$$
B\langle\mathbf{N}\rangle=\langle\mathbf{S}\rangle
$$

where $B$ is a matrix whose form depends on the physical assumptions made. The first two components of $\langle\mathbf{N}\rangle$ are the fast and slow steady state neutron densities and the remaining components are the steady-state precursor densities. The vector $\langle\mathbf{S}\rangle$ has components $\left\langle S_{\mathrm{ex}}\right\rangle \delta_{i 1}$ where $\left\langle S_{\mathrm{ex}}\right\rangle$ is the mean value of the external non-fission source assumed to emit fast neutrons only. In addition to this assumption, it is also assumed that (1) all fission neutrons, prompt as well as delayed, are fast neutrons, and (2) no fast fission is produced although fast capture may take place. The reaction rates are denoted by " $r$ " with subscripts: $f$ for fission, $s$ for scattering from fast into slow group, $t$ for total reaction rate in the fast group, $a$ for absorption in the slow group, $D_{i}, i=1,2$ for detection of fast, $i=1$, and slow, $i=2$, neutrons, and $C_{i}$, $i=1,2$ for capture in both groups.

Introducing the notation

$$
l_{1}=\frac{1}{r_{t}}, l_{2}=\frac{1}{r_{a}}, P_{12}=\frac{r_{s}}{r_{t}}, K=\langle j\rangle \frac{r_{f}}{r_{a}}
$$

the matrix B becomes:

$$
\mathrm{B}=\left(\begin{array}{cccc}
\frac{1}{l_{1}}-\frac{K}{l_{2}}(1-\beta) & -\lambda_{3} \ldots & -\lambda_{M} \\
-\frac{P_{12}}{l_{1}} \quad \frac{1}{l_{2}} & 0 & \ldots & 0 \\
0-\frac{K}{l_{2}} \beta_{3} & \lambda_{3} \ldots & 0 \\
\cdot & \cdot & & \\
\cdot & \cdot & \cdot & \\
\cdot & \cdot & & \\
0-\frac{K}{l_{2}} \beta_{M} & 0 & \ldots & \lambda_{M}
\end{array}\right)
$$


with $\langle j\rangle, \beta_{i}$ and $\lambda_{i}(i=3, \ldots, M=m+2)$ having their conventional meaning. Equation (1) can be solved for $\left\langle N_{1}\right\rangle$ and $\left\langle N_{2}\right\rangle$ yielding the following result:

$$
\begin{aligned}
& \left\langle N_{1}\right\rangle=\frac{l_{1}}{1-K P_{12}}\left\langle\mathrm{~S}_{\mathrm{ex}}\right\rangle \\
& \left\langle N_{2}\right\rangle=\frac{l_{2} P_{12}}{1-K P_{12}}\left\langle\mathrm{~S}_{\mathrm{ex}}\right\rangle .
\end{aligned}
$$

Let us define $\mathbf{n}(t)$ as a vector whose components are the deviations from $\langle\mathbf{N}(t)\rangle$ of the instantaneous values of the densities. This vector satisfies Langevin's equation (AKCASU et al., 1966)

$$
\frac{\mathrm{d}}{\mathrm{d} t} \mathbf{n}(t)=-\mathrm{Bn}(t)+\mathbf{s}(t)
$$

where $\mathbf{s}(t)$ is the noise equivalent source vector of the system. The components of $\mathbf{s}(t)$ are noise equivalent sources associated with the fluctuations in the various nuclear processes, absorption, fission, scattering, detection, decay, and emission by external source, going on in the reactor. The properties of $\mathbf{s}(t)$ will be the subject of the discussion in Section 4.

\section{DETECTION STATISTICS AND POWER SPECTRAL DENSITIES}

We assume that two detectors are present characterized by their detection rates $r_{D_{i}}(i=1,2)$. Since the reactor-detectors system is operating at steady state, the mean number of neutrons being detected per second is $r_{D_{i}}\left\langle N_{i}\right\rangle, i=1,2$. The actual number fluctuates about this mean and we postulate the existence of the following relations:

$$
z_{i}(t)=r_{D_{i}} h_{i}(t)+s_{D_{i}}(t) \quad i=1,2
$$

between $z_{i}(t)$, the instantaneous deviation of the number of neutrons detected in each detector per second and $n_{i}(t)$. The deviations of the actual output currents of the detectors about their mean are assumed to be proportional to $z_{i}(t)$. For convenience, the constants of proportionality are assumed to be unity. The noise equivalent sources $s_{D i}(t)$ are associated with the fluctuations in the detection process.

In a noise experiment, one measures $z_{i}(t), i=1,2$, as the fluctuations in the output currents of the detectors. One can compute digitally or measure directly by analog techniques, the Fourier transforms of $\left\langle z_{i}(t) z_{i}(t+\tau)\right\rangle, i=1,2$, and $\left\langle z_{i}(t) z_{2}(t+\tau)\right\rangle$ which are the auto-power and the cross-power spectral densities of the two energy groups. Since these are the observed quantities, the procedure to follow in their calculations consists of solving the stochastic equations (4) for them by eliminating the unobserved variables $n_{i}(t) i=1,2, \ldots, M$, in terms of the auto-power and crosspower spectral densities of the various noise equivalent sources.

Let us indicate by $\overline{f(\omega)}$ the Fourier transform of $f(t)$. Taking the Fourier transform of equations (3), solving for $\overline{n_{i}(\omega)}, i=1,2$, and substituting in the Fourier transformed equation (4), we can obtain expressions for $\overline{z_{i}(\omega)}, i=1,2$. The details are left for Appendix II. 
The power-spectral-densities of the output detector currents are then defined as:

$$
\Phi_{m n}(\omega)=\int_{-\infty}^{+\infty} \mathrm{d} \tau \mathrm{e}^{-i \omega \tau}\left\langle z_{m}(t) z_{n}(t+\tau)\right\rangle \quad m, n=1,2 .
$$

This is the quantity that is measured in an experiment. By writing $z_{i}(t), i=1,2$, in terms of $z_{i}(\omega)$ we can rewrite equation (6) as follows:

$$
\Phi_{m n}(\omega)=\frac{1}{2 \pi} \int_{-\infty}^{+\infty} \mathrm{d} \omega^{\prime} \exp \left[-i\left(\omega+\omega^{\prime}\right) t\right]\left\langle\overline{\left\langle z_{m}\left(\omega^{\prime}\right)\right.} \overline{z_{n}(\omega)}\right\rangle .
$$

This function depends on the stochastic properties of $\mathbf{s}(t)$, the discussion of which will be the task of the next section.

\section{STOCHASTIC PROPERTIES OF THE NOISE EQUIVALENT SOURCES}

It is convenient to write the components of $\mathbf{s}(t)$ as a sum of various noise equivalent sources representing the stochastic processes originating the fluctuations in $\mathbf{n}(t)$. This splitting up of $\mathbf{s}(t)$ is introduced to facilitate the calculation of various auto- and cross-correlation functions of noise equivalent sources in the framework of certain assumptions. With this in mind we write:

$$
s_{1}(t)=s_{2}{ }^{F G}(t)-s_{1}{ }^{c}(t)-s_{1}{ }^{S}(t)-s_{D_{1}}(t)+s_{\mathrm{ex}}(t)+\sum_{l=3}^{m} \mathrm{~d}_{e}(t) .
$$

and

$$
\begin{aligned}
& s_{2}(t)=-s_{2}{ }^{F L}(t)+s_{1}^{S}(t)-s_{2}^{c}(t)-s_{D_{2}}(t) \\
& s_{l}(t)=p_{l}(t)-d_{l}(t) \quad l=3, \ldots, M .
\end{aligned}
$$

The minus sign in front of some of the noise-equivalent sources indicates that they are associated with processes that produce a loss in the number of neutrons. The superscripts $F G$ and $F L$ mean fission gain and fission loss, that is the noise equivalent sources carrying those superscripts are associated with the fluctuations in the gain of fission neutrons in the fast group and with the fluctuations in the number of neutrons lost in the slow group due to a fission absorption, respectively. The remaining superscripts have the same meaning as in the subscripts for the reaction rates. Finally the noise equivalent source $p_{l}(t)$ is associated with the production rate of delayed neutron precursors of the $l$-th type and $d_{l}(t)$ with the decay of the said precursor. Some assumptions are necessary to proceed further in the study of the noise equivalent sources. First it is assumed that the random fluctuations in the number of events of capture in both energy groups, scattering, fission, and decay of delayed neutrons precursors are independent of one another. This implies that in our terminology, only cross correlations exist among $s_{2}{ }^{F G}(t), s_{2}{ }^{F L}(t)$ and $p_{t}(t)$. Second, the probability per unit time of occurrences of each event mentioned in the above assumption is constant in time. Third, the fluctuations in the number of events of a given kind in two non-overlapping time intervals are statistically independent. And fourth, in any fission not more than one delayed neutron precursor is produced. In view of these assumptions and following Akcasu and Osborn (1966), the correlation functions for 


\begin{tabular}{|c|c|c|c|}
\hline$i \backslash k$ & $C$ & $S$ & $D$ \\
\hline $\begin{array}{l}1 \\
2\end{array}$ & $\begin{array}{l}r_{r_{1}} \\
r_{c_{2}}\end{array}$ & $r_{s}$ & $\begin{array}{l}r_{D_{1}} \\
r_{D_{2}}\end{array}$ \\
\hline
\end{tabular}

\begin{tabular}{cccc} 
TARLE $2 .-B_{f}{ }^{g}, l, k=3,4, \ldots M\langle f(t) g(t+\tau)\rangle=B_{f}{ }^{g}\left\langle N_{2}\right\rangle \delta(\tau)$ \\
\hline$p_{\imath}$ & $s_{2}{ }^{F G}$ & $s_{2}{ }^{F L}$ & $f l g$ \\
\hline$\delta_{l k}\langle j\rangle \beta_{l} r_{f}$ & $\left\langle j_{p}\right\rangle\langle j\rangle \beta_{k} r_{f}$ & $\langle j\rangle \beta_{k} r_{f}$ & $p_{k}$ \\
$\left\langle j_{p}\right\rangle\langle j\rangle \beta_{l} r_{f}$ & $\left\langle j_{p}{ }^{2}\right\rangle r_{f}$ & $\left\langle j_{p}\right\rangle r_{f}$ & $s_{2}{ }^{F G}$ \\
$\langle j\rangle \beta_{l} r_{f}$ & $+\left\langle j_{p}\right\rangle r_{f}$ & $r_{f}$ & $s_{2}{ }^{F L}$ \\
\hline
\end{tabular}

the different noise equivalent sources can be easily obtained. The results are given in Tables I and II and equations $9 \mathrm{ab}$.

$$
\begin{aligned}
\left\langle s_{\mathrm{ex}}(t) s_{\mathrm{ex}}(t+\tau)\right\rangle & =\left\langle s_{\mathrm{ex}}\right\rangle \delta(\tau) \\
\left\langle d_{l}(t) d_{k}(t+\tau)\right\rangle & =\delta_{l k}\langle j\rangle \beta_{l} r_{f}\left\langle N_{2}\right\rangle \delta(\tau) .
\end{aligned}
$$

In Tables 1 and 2 the subindexes 1 and 2 refer to fast and slow groups respectively and the subindex $p$ in $\left\langle j_{p}\right\rangle$ and $\left\langle j_{p}{ }^{2}\right\rangle$ refers to prompt neutrons. It should be noted that the result given in these tables and equations $(9 \mathrm{ab})$ obtained by application of the Langevin technique, could have been obtained directly using the fact that the time evolution of the neutron population in a reactor is a Markoff random process.

It is easily seen that to obtain ensemble averages of the form $\left\langle\overline{s(\omega)} \overline{s\left(\omega^{\prime}\right)}\right\rangle$ one merely replaces $\delta(\tau)$ by $2 \pi \delta\left(\omega+\omega^{\prime}\right)$ in equations (9). For instance:

$$
\overline{\left\langle s_{1}^{c}(\omega) \overline{S_{1}^{c}}\left(\omega^{\prime}\right)\right\rangle}=r_{c_{1}}\left\langle N_{1}\right\rangle 2 \pi \delta\left(\omega+\omega^{\prime}\right)
$$

A relation between $\left\langle j_{p}{ }^{2}\right\rangle$ and $\left\langle j^{2}\right\rangle$ was developed by AKCASU et al. (1966):

$$
\left\langle j^{2}\right\rangle=\left\langle j_{p}{ }^{2}\right\rangle+2\langle j\rangle^{2} \beta(1-\beta)+\langle j\rangle \beta
$$

which is based on two physical assumptions: (a) the number of prompt neutrons produced in any fission event is independent of the production of delayed neutrons and (b) not more than one delayed neutron is produced in any fission event. Other relations can be found in the literature based on a set of different assumptions. (OTSUKA et al., 1965)

\section{ANALYTICAL EXPRESSIONS FOR THE SPECTRAL DENSITIES}

As outlined in Section 2, once expressions for $\overline{z_{i}(\omega)}$ are obtained, substitution into equations (7) yields analytical formulas for $\Phi_{11}, \Phi_{22}$, and $\Phi_{12}$. The results for a general case are given in Appendix 2. If we consider one delayed group only and notice that quite generally $\lambda\left(l_{1}+l_{2}\right) \ll 1, \lambda \ll r a_{2}, l_{2} \ll l_{1}$, the spectral densities can be written in a simple form:

$$
\begin{aligned}
\Phi_{11}(\omega) & =A_{11} D(\omega)\left(\omega^{2}+\omega_{4}^{2}\right)+C_{11} \\
\Phi_{22}(\omega) & =A_{22} D(\omega)+C_{22} \\
\operatorname{Re} \Phi_{12}(\omega) & =A_{12} D(\omega) \\
\operatorname{Im} \Phi_{12}(\omega) & =A_{21} \omega D(\omega)
\end{aligned}
$$


where

$$
D(\omega)=\left(\omega^{2}+\omega_{5}\right)^{2}\left(\omega^{2}+\omega_{1}^{2}\right)^{-1}\left(\omega^{2}+\omega_{2}^{2}\right)^{-1}\left(\omega^{2}+\omega_{3}^{2}\right)^{-1} .
$$

The break frequencies and the constants in equations (10) are given below in Table 3.

TABLE 3.-TWO GROUP CONSTANTS AND BREAK FREQUENCIES

\begin{tabular}{|c|c|}
\hline$A, B$ & $\omega$ \\
\hline$A_{11}=r_{D_{1}}^{2} q$ & $\omega_{1}^{2}=r_{t}^{2}$ \\
\hline$C_{11}=r_{D_{1}}\left\langle N_{1}\right\rangle$ & $\omega_{2}^{2}=\left[1-K P_{12}(1-\beta)\right]^{2} r_{a}^{2}$ \\
\hline$A_{23}=r_{D_{2}}{ }^{2} r_{t}^{2} P_{12}^{2} q$ & $\omega_{3}{ }^{2}=\left(1-K P_{12}\right)^{2}\left[1-K P_{12}(1-\beta)\right]^{-2} \lambda^{2}$ \\
\hline$C_{22}=r_{D_{2}}\left\langle N_{2}\right\rangle$ & $\omega_{4}^{2}=r_{a}^{2}$ \\
\hline$A_{21}=r_{D_{1}} r_{D_{2}} r_{t} r_{a} P_{12} q$ & $\omega_{s}^{2}=r_{f}\left\langle N_{2}\right\rangle\langle j(j-1)\rangle q^{-1} \lambda^{2}$ \\
\hline $\begin{aligned} A_{12} & =-r_{D_{1}} r_{D_{2}} r_{t} P_{12} q \\
q & =r_{f}\left\langle N_{2}\right\rangle\left[\langle j(j-1)\rangle-2\langle j\rangle^{2} \beta(1-\beta)\right]\end{aligned}$ & \\
\hline
\end{tabular}

Letting $l_{1}=0$ and $P_{12}=1$, we obtain from $\Phi_{22}$ the one-group P.S.D.:

$$
\Phi_{20}(\omega)=A_{0}\left(\omega^{2}+{\omega_{5}^{2}}^{2}\right)\left(\omega^{2}+{\omega_{02}}^{2}\right)^{-1}\left(\omega^{2}+\omega_{03}^{2}\right)^{-1}+B_{0} .
$$

The new constants and break frequencies are given below in Table 4 .

TABLE 4.-ONE-GROUP CONSTANTS AND BREAK FREQUENCIES

\begin{tabular}{ll}
\hline \multicolumn{1}{c}{$A_{0}, B_{0}$} & $\omega$ \\
\hline$A_{0}=r_{D}{ }^{2} \varphi$ & $\omega_{02}{ }^{2}=[1-K(1-\beta)]^{2} r_{a}{ }^{2}$ \\
$C_{0}=r_{D}\langle N\rangle$ & $\omega_{03}{ }^{2}=(1-K)^{2}[1-K(1-\beta)]^{-2} \lambda^{2}$ \\
\hline
\end{tabular}

A comparison of Tables 3 and 4 shows that a two-group model provides the same information as the one group model if one replaces $\mathrm{K}$ by $\mathrm{KP}_{12}$. In addition to this the two-group model used in this paper predicts direct observation of the fast and slow neutron life-times. All the break frequencies contained in the slow group P.S.D. and in the C.P.S.D. are contained in the fast group P.S.D. However, the fast group P.S.D. contains an additional frequency, namely $r_{a 2}$. Therefore, a measurement of $\Phi_{11}$ will yield all the information in all the spectral densities. The phase of the C.P.S.D. obeys the simple rule $\tan \theta_{12}=-\omega l_{2}$ which suggests an alternate way of measuring $l_{2}$. A measurement of either the real part or the imaginary part of the C.P.S.D. will remove the so called detector noise which is known to be an advantage from an experimental point of view.

\section{NUMERICAL APPLICATIONS}

The values of the parameters used in this numerical application are given in Table 5 (THIE, 1963; ANL-5800, 1963). The order of magnitude of the parameters chosen represents a thermal reactor operating at low power level and has low resonance absorption. The numerical value of $\left\langle S_{\mathrm{ex}}\right\rangle$ was chosen arbitrarily to yield $\left\langle N_{1}\right\rangle=10$ neuts $/ \mathrm{cm}^{3}$ which simplifies the numerical calculations. The results are given in Table 6. Graphs for $\Phi_{11}$ and $\tan \theta_{12}$ are given in Figs. 1 and 2. Very low and high frequencies which lie outside the range of practical interest are also included in the graphs for the sake of completeness. 
TABLE 5.- TWO GROUP PARAMETERS

\begin{tabular}{rlrl}
\hline$P_{12}$ & $=0.995$ & $\beta$ & $=7.5 \times 10^{-3}$ \\
$K$ & $=0.99$ & $r_{b_{1}}$ & $=3.5 \times 10^{3} \mathrm{sec}^{-1}$ \\
$l_{1}$ & $=7.8 \times 10^{-8} \mathrm{sec}$ & $r_{D_{1}}$ & $=6.8 \times 10^{2} \mathrm{sec}^{-1}$ \\
$l_{2}$ & $=10^{-4} \mathrm{sec}$ & $r_{f}$ & $=3.9 \times 10^{3} \mathrm{sec}^{-1}$ \\
$\langle j\rangle$ & $=2.5$ & $\left\langle N_{1}\right\rangle$ & $=10$ neuts-cm \\
$\left\langle j\left(j^{-1}\right)\right\rangle$ & $=4.97$ & $\left\langle N_{2}\right\rangle$ & $=1.3 \times 10^{4}$ neuts-cm \\
$\lambda$ & & $\left\langle S_{\mathrm{ex}}\right\rangle$ & $=1.9 \times 10^{6}$ neuts-cm \\
\hline
\end{tabular}

Table 6.-Numerical Values of TWO-Group Spectral DeNSITY CONSTANTS AND BREAK FREQUENCIES

\begin{tabular}{ll}
\hline$A_{11}=3 \times 10^{15} \mathrm{sec}^{-3} \mathrm{~cm}^{-3}$ & $\omega_{1}=1.3 \times 10^{7} \mathrm{sec}^{-1}=r_{t}$ \\
$A_{22}=1.4 \times 10^{28} \mathrm{sec}^{-5} \mathrm{~cm}^{-3}$ & $\omega_{2}=2.2 \times 10^{2} \mathrm{sec}^{-1}=2.2 \times 10^{-2} \times r t$ \\
$A_{12}=7.4 \times 10^{25} \mathrm{sec}^{-5} \mathrm{~cm}^{-3}$ & $\omega_{3}=5.1 \times 10^{-2} \mathrm{sec}^{-1}=0.7 \times \lambda$ \\
$A_{21}=7.4 \times 10^{21} \mathrm{sec}^{-4} \mathrm{~cm}^{-3}$ & $\omega_{4}=10^{4} \mathrm{sec}^{-1}=r_{a}$ \\
$B_{11}=3.4 \times 10^{4} \mathrm{sec}^{-1} \mathrm{~cm}^{-8}$ & $\omega_{5}=7.68 \times 10^{-2} \mathrm{sec}{ }^{1}=1.01 \times \lambda$ \\
$B_{22}=8.6 \times 10^{6} \mathrm{sec}^{-1} \mathrm{~cm}^{-3}$ & \\
\hline
\end{tabular}

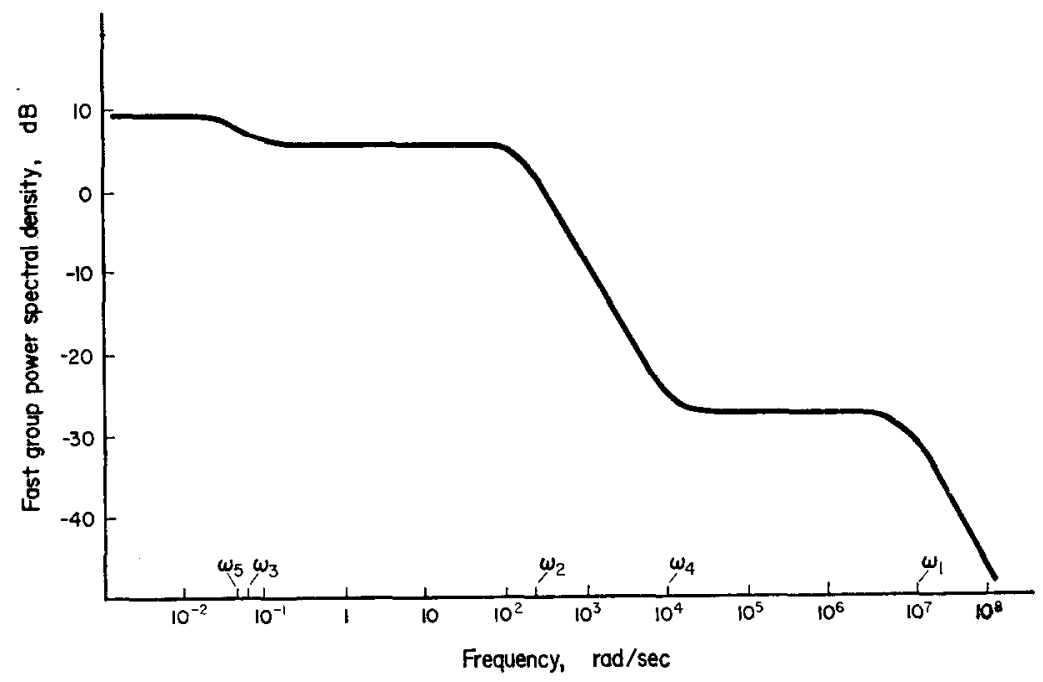

FIG. 1.-_Fast-group power spectral density (db) vs. frequency (rad./sec).

The break frequencies introduced due to the inclusion of delayed neutrons in the analysis are seen to be proportional to $\lambda$. In the graph for $\Phi_{11}$, they are noticeable as a ripple in the low frequency range. If the C.P.S.D. $\Phi_{12}$ is written in the form: $\Phi_{12}=A(\omega) \exp \left[\theta_{12}(\omega)\right]$ where $A(\omega)$ is the gain and $\theta_{12}(\omega)$ the phase angle, the following relation is found to be valid for all frequencies:

$$
\tan \theta_{12}(\omega)=-\omega l_{2}
$$

which suggests a way of measuring $l_{2}$ directly.

\section{CONCLUSIONS AND DISCUSSION}

Langevin's technique has been applied to a two energy group, space independent model and explicit expressions for the auto- and cross-power spectral densities of the 
fast and slow groups have been obtained. The correlations due to fluctuations in production and decay of delayed neutron precursors, which are not included in the paper by CoHN (1964) are also taken into account. They are, however, shown to be proportional to the delayed neutron fraction $\beta$ and are observed to be small as compared to other correlations. The correlation between the fluctuation sources in the fast and slow group is observed to be not only due to fluctuations in fission process but also due to random nature of scattering from fast to slow group. The general results agree with those given in a paper by SAITo (1967b).

The analysis yields additional break frequencies, as compared to the one group case, some of which could be used for measuring certain group constants and reactor

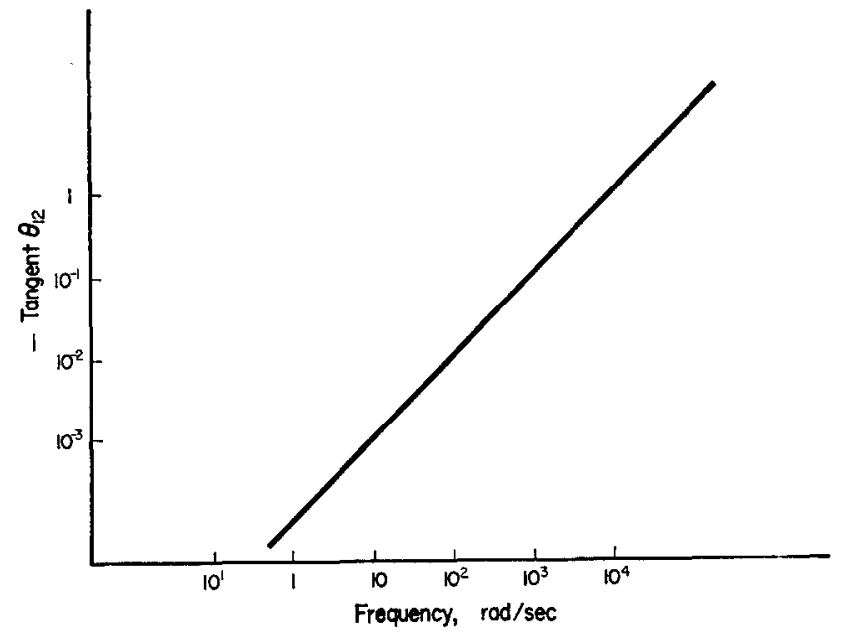

Fig. 2.-Negative of the tangent of the phase angle of the cross-power spectral density vs. frequency (rad./sec).

parameters. It is observed that information contained in the break frequencies of the slow group P.S.D. in the two group analysis is essentially the same as that resulting from the break frequencies in one group analysis in which $K$ is modified to include resonance escape probability. The information contained in the P.S.D. of the fast group is the same as that in the P.S.D. of the slow group except for the frequency $l_{2}{ }^{-1}$ which is not directly observable in the slow group P.S.D. This frequency may also be obtained from a measurement of the phase of $\Phi_{12}$ which obeys the simple rule: $\tan \theta_{12}(\omega)=-\omega l_{2}$.

The results of this paper assumed a space independent model as well as a detector of infinite size. The influence of the finiteness of both medium and detector has been the subject of considerable discussion in papers by NATELSON et al. (1966), SHEFF et al. (1966a; 1966b) and more recently by WiLliams (1967). According to the latter author, for an infinite medium, the size and geometry of the detector greatly affects the shape of the P.S.D. Only for detectors many diffusion lengths in size, the P.S.D. becomes the conventional one of the infinite medium, infinite detector case. For finite media, the influence of the detector shape on the functional form of the P.S.D. is not very strong, and the smaller the reactor, the less strong this influence is, provided measurements are made in a restricted frequency range. For large systems, the reverse effect occurs, so that direct application of the infinite medium model may be 
more efficient. In view of these remarks, the results of this paper might be applicable to either a very small reactor or to a very large one with large detectors.

Finally, from a mathematical point of view, the application of Langevin's technique to more complicated models where many energy groups are considered is straight forward. This is due to the fact that the stochastic equations resulting from its application are the same in number as the equations for averaged quantities describing the system, an advantage which is not demonstrated in other techniques (MATTHeS, 1966; NATELSON et al., 1966) recently developed for noise analysis. As shown in the present analysis, the application of Langevin's technique to a multigroup description involves noise equivalent sources in each group whose correlations can easily be obtained from simple physical considerations. All of the new break frequencies arising from the multigroup description may not be physically observable in general if the detector samples only the thermal group. In the two group treatment in which detectors respond to thermal and fast groups, one can expect distinct break frequencies in the measured P.S.D. which can be related unambiguously to the life times associated with each group. In this case, the division of the energy spectrum into two groups is not only a mathematical convenience but it also has a physical origin since the detectors are assumed to be sensitive to neutrons in each group.

\section{REFERENCES}

ANL-5800 (1963) Reactor Physics Constants.

AKCAsu A. Z. and Osborn R. K. (1966) Nucl. Sci. Engng 26, 13.

BADGley R. W. and Uhrig R. E. (1957) Nucl. Sci. Engng 2, 450.

BeLl G. I. (1965) Nucl. Sci. Engng 21, 390.

BenNetT E. F. (1960) Nucl. Sci. Engng 8, 53.

CoHN C. E. (1960) Nucl. Sci. Engng 7, 472.

CoHN C. E. (1964) Report TID-7679, 307.

Courant E. D. and Wallace P. R. (1947) Phys. Rev. 72, 1038.

DALFES A. (1962) Nukleonik 4, 299.

De Hoffman F. (1949) The Science and Engineering of Nuclear Power, C. D. Goodman Ed., AddisonWesley Press, Vol. II, 103.

DraGT J. B. (1966) Nukleonik 8, 188.

GoToн S. (1966) J. Nucl. Sci. Technol. 3, 359.

HARRIS D. R. (1958) Report WAPD-TM-190,

KLAHR C. N. (1958) Nucl. Sci. Engng 3, 269.

Langevin M. P. (1908) C.r. hebd. Séanc Acad. Sci., Paris 146, 530.

LAX M. (1960) Rev. mod. Phys. 32, 25.

MATTHES W. (1966) Nukleonik 8, 87.

MOORE M. N. (1958) Nucl. Sci. Engng 6, 448.

Natelson M., Osborn R. K. and Shure F. (1966) J. Nucl. Energy Parts A/B 20, 557.

Nomura T. (1966) J. Nucl. Sci. Technol. 3, 14.

ORNDOFF J. D. (1957) Nucl. Sci. Engng 2, 450.

OSBORN R. K. (1965) Report ORNL-3757.

OSBORN R. K. and NIETo J. M. (1966) Nucl. Sci. Engng 26, 511 .

OsboRn R. K. and YIP S. (1963) Trans. ANS 6, No. 1.

Otsuka M. and Sarto K. (1965) J. Nucl. Sci. Technol. 2, 191.

PaL L. (1958) Nuovo Cim. Vol. VII (Supplement), Serie X, No. 1, 25.

RAIEvSKI V. (1958) Report CEA 917.

SAITO K. (1967a) Nucl. Sci. Engng 28, 384.

SAITo K. (1967b) Nucl. Sci. Engng 28, 452.

ShefF J. R. and Albrecht R. W. (1966a) Nucl. Sci. Engng 24, 246.

SheFF J. R. and Albrecht R. W. (1966b) Nucl. Sci. Engng 26, 207.

THIE J. A. (1963) Reactor Noise (AEC Monograph), Rowman and Littlefield, New York.

Trinko J. R. JR. and Hanauer S. (1965) Trans ANS 8, No. 1.

Velez C. (1959) Nucl. Sci. Engng 6, 414.

Williams M. M. R. (1967) J. Nucl. Energy 21, 321. 


\section{APPENDIX 1}

Let us consider a reactor model with $n$ energy groups and $m$ delayed neutron precursors. The vector $\mathbf{n}(t)$ has then, $n+m$ components. Solving equation (3) formally for the column vector $\mathbf{n}(t)$ gives:

$$
\mathbf{n}(t)=\int_{0}^{\infty} \mathrm{e}-u B \mathrm{~s}(t-u) \mathrm{d} u .
$$

Defining row vectors $\mathbf{n}^{T}(t)$ and $\mathbf{s}^{T}(t)$, and the noise equivalent source spectral matrix $2 D$ by (SAITo, 1967a):

$$
\left\langle\mathbf{s}(t) \mathbf{s}^{T}(t+\tau)\right\rangle=2 D \delta(\tau)
$$

equation (I-2) gives:

$$
\Phi_{n}(\tau)=\left\langle\mathbf{n}(t) \mathbf{n}^{T}(t+\tau)\right\rangle=\int_{0}^{\infty} \int_{0}^{\infty} \mathrm{d} u \mathrm{~d} v \mathrm{e}^{-u B} 2 D e^{-v B^{T}} \delta(\tau+u-v)
$$

where $B^{T}$ is the transposed of $B$. Following Saito (SAIto, 1967a), we make use of the generalized Schottky formula (CoHN, 1960)

$$
2 D=Q+B E+E B^{T}
$$

where $E$ is a diagonal matrix whose elements are $\left\langle N_{i}\right\rangle, i=1,2, \ldots, m+n$, and $Q=B V+V B^{r}$ where $V$ is the modified covariance matrix defined by Saito (SAITo, 1967a).

From equations (I-2) and (I-4) one obtains $\Phi_{n}(\tau)$ as the sum of two terms:

$$
\Phi_{n}(\tau)=\int_{0}^{\infty} \int_{0}^{\infty} \mathrm{d} u \mathrm{~d} v \mathrm{e}^{-u B} Q e^{-v B^{T}} \delta(\tau+u-v)+\left\{\begin{array}{l}
E \mathrm{e}^{-\tau B^{T}}, \tau>0 \\
\mathrm{e}^{-|\tau| B} E, \tau<0
\end{array}\right\} .
$$

This equation is easily Fourier transformed giving:

where:

$$
G_{n}(\omega)=G(-\omega) Q G^{T}(\omega)+E G^{T}(\omega)+G(-\omega) E
$$

$$
G(\omega)=\int_{0}^{\infty} \mathrm{e}^{-u B} \mathrm{e}^{-i \omega u} \mathrm{~d} u
$$

is the Laplace-Fourier transform of the Green's function $\mathrm{e}^{-u B} . G_{n}(\omega)$ is the neutron-precursor power spectral density matrix (SAITo, 1967a).

Suppose now that we have $n$ detectors characterized by their reaction rates $r_{D_{i}}, i=1,2, \ldots, n$.

We postulate the stochastic equation:

$$
\mathrm{z}(t)=R \mathbf{n}(t)+\mathrm{s}_{D}(t)
$$

where $R$ is a diagonal matrix whose diagonal elements are $r_{D_{i}}, i=1,2, \ldots, n$, the remaining ones being identically zero. The vector $\mathbf{s}_{D}(t)$ is the noise equivalent source vector associated with the fluctuations in the detection process and its only non-vanishing components are $s_{D_{i}}(t), i=1,2, \ldots$, $n$. Thus $\mathbf{z}(t)$ has only non-vanishing components $z_{i}(t)$ for $i=1,2, \ldots, n$.

It is easy to see that (AKCASU et al., 1966)

$$
\left\langle\mathbf{s}(t) \mathbf{s}_{D}{ }^{T}(t+\tau)\right\rangle=\left\langle\mathbf{s}_{D}(t) \mathbf{s}^{T}(t+\tau)\right\rangle=-\Delta \delta(\tau)
$$

where $\Delta$ is a diagonal matrix whose diagonal elements are $r_{D_{i}}\langle\mathrm{Ni}\rangle, i=1,2, \ldots, n$, the remaining ones being zero. From equations (I-7) and (I-1) one obtains immediately:

$$
\begin{aligned}
& \Phi_{z}(\tau)=\left\langle\mathbf{z}(t) \mathbf{z}^{T}(t+\tau)\right\rangle=R \Phi_{n}(\tau) R+\Delta \delta(\tau) \\
& -R\left\{\begin{array}{cc}
0, & \tau>0 \\
\mathrm{e}-|\tau| B \Delta, & \tau<0
\end{array}\right\}-\left\{\begin{array}{cc}
\Delta \mathrm{e}^{-\tau B}, \tau>0 \\
0, & \tau<0
\end{array}\right\} R .
\end{aligned}
$$

Fourier transforming equation (I-8), we finally get:

$$
G_{z}(\omega)=R G_{n}(\omega) R-R G(-\omega) \Delta-\Delta G^{T}(\omega) R+\Delta
$$

This formula is the counterpart in matrix form, of equation (70) given by ACKASU and OSBORN (1966) in the space and energy dependent case. 


\section{APPENDIX 2}

Equation (3) is equivalent to the following set of equations:

$$
\begin{aligned}
\frac{\mathrm{d}}{\mathrm{d} t} n_{1}(t) & =\frac{K}{l_{2}}(1-\beta) n_{2}(t)-\frac{1}{l} n_{1}(t)+\sum_{l=3}^{M} n_{l}(t)+s_{1}(t) \\
\frac{\mathrm{d}}{\mathrm{d} t} n_{2}(t) & =\frac{P_{12}}{l_{1}} n_{1}(t)-\frac{1}{l_{2}} n_{2}(t)+s_{2}(t) \\
\frac{\mathrm{d}}{\mathrm{d} t} n_{l}(t) & =\frac{K}{l_{2}} \beta_{l} n_{2}(t)-\lambda_{l} n_{l}(t)+P_{l}(t)-\mathrm{d}_{l}(t) \quad l=3, \ldots, M .
\end{aligned}
$$

Fourier transforming these equations, solving for $n_{i}(\omega), i=1,2$ and substituting into the Fourier transformed equations (4), gives:

where

$$
\begin{aligned}
& \left.\overline{z_{1}(\omega)}=\frac{l_{1} r_{D_{1}}}{\Delta(i \omega)}\left\{\overline{\left[s_{1}(\omega)\right.}+\overline{s_{d}(\omega)}\right]\left(1+i \omega l_{2}\right)+\overline{s_{2}(\omega)} K g(i \omega)\right\}+\overline{s_{D_{1}}(\omega)} \\
& \left.\overline{z_{2}(\omega)}=\frac{l_{2} r_{D_{2}}}{\Delta(i \omega)}\left\{\overline{\left[s_{1}(\omega)\right.}+\overline{s_{a}(\omega)}\right] P_{12}+\overline{s_{2}(\omega)}\left(1+i \omega l_{1}\right)\right\}+\overline{s_{D_{2}}(\omega)}
\end{aligned}
$$

Defining

$$
\begin{aligned}
& \Delta(i \omega)=-\omega^{2} l_{1} l_{2}+i \omega\left(l_{1}+l_{2}\right)+1-K P_{12} g(i \omega) \\
& g(i \omega)=1-F(i \omega) ; \quad F(i \omega)=\sum_{l=3}^{M} \frac{\beta_{l} i \omega}{i \omega+\lambda_{l}} \\
& \overline{s_{d}(i \omega)}=\sum_{l=3}^{M} \frac{i \omega \overline{d_{l}(\omega)}+\lambda_{l} P_{l}(\omega)}{i \omega+\lambda_{l}} .
\end{aligned}
$$

$$
\begin{gathered}
S_{11}=\left\langle j_{p}{ }^{2}\right\rangle r_{f}\left\langle N_{z}\right\rangle+r_{t}\left\langle N_{1}\right\rangle+\left\langle S_{\mathrm{ex}}\right\rangle \\
S_{22}=r_{a}\left\langle N_{2}\right\rangle+r_{s}\left\langle N_{1}\right\rangle \\
S_{12}=-\left\langle j_{p}\right\rangle r_{l}\left\langle N_{2}\right\rangle-r_{s}\left\langle N_{1}\right\rangle \\
S_{d d}=+\langle j\rangle \beta\left\langle N_{2}\right\rangle r_{f} \\
\left.S_{1 d}(\omega)=\sum_{l=3}^{M} \frac{\lambda_{l}}{\lambda_{l}-i \omega}\left\langle j_{p}\right\rangle j\right\rangle \beta_{l} r_{f}\left\langle N_{2}\right\rangle \\
S_{2 d}(\omega)=-\sum_{l=3}^{M} \frac{\lambda_{l}}{\lambda_{l}-i \omega}\langle j\rangle \beta_{l} r_{f}\left\langle N_{2}\right\rangle \\
A(\omega)=K S_{12} g(i \omega)\left(1-i \omega l_{2}\right)+s_{1 d}(\omega)\left(1+\omega^{2} l_{2}{ }_{2}\right)+K S_{2 d}(\omega) g(i \omega)\left(1-i \omega l_{2}\right) \\
-\frac{\left\langle N_{1}\right\rangle}{l_{2}}\left(1+i \omega l_{2}\right) \Delta^{*}(i \omega) \\
B(\omega)=P_{12}{ }^{2} S_{1 \dot{ }}(i \omega)+P_{12} S_{2 d}(\omega)\left(1+i \omega l_{1}\right)-\frac{\left\langle N_{2}\right\rangle}{l_{2}}\left(1+i \omega l_{1}\right) \Delta^{*}(i \omega)
\end{gathered}
$$

where ${ }^{*}$ means complex conjugate, the spectral densities can be written as follows:

$$
\begin{aligned}
\Phi_{11}(\omega)= & \frac{l_{1}{ }^{2} r_{D 1}{ }^{2}}{|\Delta(i \omega)|^{2}}\left\{\left(S_{11}+S_{d d}\right)\left(1+\omega^{2} l_{2}^{2}\right)+S_{22} K^{2}|g(i \omega)|^{2}+2 \operatorname{ReA}(\omega)\right\}+r_{D_{1}}\left\langle N_{1}\right\rangle \\
\Phi_{22}(\omega)= & \frac{l_{2}^{2} r_{D_{2}}{ }^{2}}{|\Delta(i \omega)|^{2}}\left\{\left(S_{11}+S_{d d}\right) P_{12}{ }^{2}+S_{22}\left(1+\omega^{2} l_{1}{ }^{2}\right)+2 P_{12} S_{12}+2 \operatorname{ReB}(\omega)\right\}+r_{D_{2}}\left\langle N_{2}\right\rangle \\
\Phi_{21}(\omega)= & \frac{l_{1} l_{2} r_{D_{1}} r_{D_{2}}}{|\Delta(i \omega)|^{2}}\left\{P_{12}\left(1+i \omega l_{2}\right)\left[S_{11}+S_{d l}+2 \operatorname{Re} S_{1 d}(\omega)\right]+S_{22} K g(i \omega)\left(1-i \omega l_{1}\right)\right. \\
& +P_{12} K g(i \omega)\left[S_{12}+S_{2 d}(\omega)\right]+\left(1+i \omega l_{2}\right)\left(1-i \omega l_{1}\right)\left[S_{12}+S_{2 d} *(\omega)\right] \\
& \left.-\frac{\left\langle N_{2}\right\rangle}{l_{2}} K g(i \omega) \Delta^{*}(i \omega)-\frac{\left\langle N_{1}\right\rangle}{l_{1}} P_{12} \Delta(i \omega)\right\}
\end{aligned}
$$

